\title{
Refugees and Trauma
}

\author{
Yichen Dong $^{1}$ \\ ${ }^{1}$ Flinders University, Adelaide, South Australia \\ Correspondence: Yichen Dong, Flinders University, Adelaide, South Australia.
}

Received: October 25, 2019

Accepted: November 15, 2019

Online Published: December 5, 2019

doi:10.20849/ajsss.v4i4.675

URL: https://doi.org/10.20849/ajsss.v4i4.675

\begin{abstract}
This essay explains the relationship between refugees and trauma. Based on the statistics of refugees in the past, this essay demonstrates why their experiences made them get trauma. Then, this essay finds out how to work with refugees from building safe working environment, rebuilding social networks, teaching them how to deal with negative emotions as well as using strength-based theory and empowerment theory. Then, some possible treatments are introduced, such as cognitive-behaviour therapy, psychoeducation and parenting intervention as well as art therapy.
\end{abstract}

Keywords: refugees, trauma, intervention, treatment

\section{Introduction}

Refugees are a group of people who has been leaved their own countries and could not be able to return their own countries safely, which is the result of various reasons, including war, race, religion, nationality, membership of a specific social group as well as political opinion (Dalton-Greyling and Campus, 2008). Due to their miserable and torturous experience, they are viewed as a specific group of people with high incidence of trauma. Therefore, this essay will explain the reasons of examining their experience, find out some factors that contribute to their trauma as well as clarify some issues which should be considered when working with them. Meanwhile, possible intervention approaches and clear reflection will be provided.

\section{Body}

\subsection{The Importance to Examine Refugees' Traumatic Experience}

Basically, social workers and related professionals could find more reasonable treatments from examining refugees' traumatic experience. This is because their experience is special while some emotional issues may be developed from their experience.

Firstly, the quantity of refugees is huge tremendously around the world, which can not be ignored. According to statistics, there were more than 65 million refugees in 2017, which was caused by violence, war, climate change, human rights deprivation as well as regional conflicts (Coats, 2017). As for Australia, more than 17,500 refugees were accepted from 2015 to 2016 (Karlsen, 2016). Syria and Iraq were two main countries of origin in these two years (Karlsen, 2016). This could lead to extremely hard settlement challenges for Australian government. In detail, some basic tasks should be solved for these refugees, such as language and cultural barriers, housing problem, employment issue as well as medical treatment and mental health. Meanwhile, some people think that accepting refugees are financial and political burdens to some extent. For example, according to the outcome of opinion polls held by the Parliamentary Library, more the more Australians insisted that refugees using boats to Australia should be regarded as an issue and deserve public concern (Phillips and Spinks, 2013). In contrast, the positive impacts of refugees can not be ignored. Specifically, they could alleviate serious shortage of the labour force, stimulate economic development as well as prosper the rural areas of Australia (Correa-Velez, Gifford and Barnett, 2010). According to statistics, refugees took a lot of jobs in farming industry, manual jobs as well as seasonal jobs, such as picking fruits. These jobs require a lot of labour force. However, aging population and underpopulation are serve so that there are not enough local people to stimulate the development of basic industries (Loomes and McCarthy, 2011).

Secondly, traumatic experience will lead to negative impacts on refugees' mental health. This is a fact that refugees could have an extremely high possibility of getting mental health problems, such as trauma and post-traumatic stress disorder (Momartin, Silove, Manicavasagar and Steel, 2004). This is the result of their 
grievous experience, from torture, displacement to any difficulties of start a new life in a new country. During this process, possible mental effects may be developed due to trauma and post-traumatic stress disorder, such as fear, sadness, anxiety and anger. These negative emotions may lead to more serious emotional issues, such as depression and suicidal tendency (Ferrada-Noli and Sundbom, 1996). For example, all asylum seekers arriving Australia without a valid visa will be viewed as unauthorised arrivals while they will be detained until they are given a valid visa or leave Australia, which is called mandatory detention (Dudley, 2003). Many researches have shown that the process of detention could be regarded as a trigger for these refugees to recall their previous traumatic experience (Smith, 1998). This could aggravate their negative emotions and feelings for their present situations and even the further life in detention centre. In detail, some negative feelings are developed due to the tension, stress as well as helplessness of the life in detention centre, such as fear, anxiety, depression as well as trauma. With more time they spent in the detention centre, more signs and symptoms of these negative emotional issues will be found (Dembo, La Voie, Schmeidler and Washburn, 1987). Another example is the process after detention, which is called resettlement. This is because for refugees, it is difficult for them to restart a new life. In detail, some refugees lost their family members so that there is a lack of social supports. At the same time, they always live dispersedly in a new country so that they can not live with people who have similar cultural background in general. This may lead to the feeling of aloneness. Moreover, it is difficult for them to find a new stable job because they cannot get a certificate of any professional qualification at a short period as a result of language barriers (De Vroome and Van Tubergen, 2010). This could lead to they may be at a lower socioeconomic status in the new country compared with their home country (Kok, 1989). Thus, some potential negative emotions may be developed, such as anxiety, sense of loss as well as grief. If they could not receive reasonable intervention, these negative emotions may lead to more serious mental health problems.

In general, refugees can not be ignored because of their huge quantity. They could bring impacts to their new country positively and negatively at the same time. Thus, it is important to examine their traumatic experience in order to eliminate its negative impacts, such as mental health issues.

\subsection{Factors for the Traumatic Nature of Refugees' Experiences}

Different culture could be one factor, which contributes to the traumatic situation of refugees. To some extent, culture could define how we understand and interact with the environment, such as thoughts, beliefs as well as behavioural modes (Ross, 2012). A person's culture is never decided by race, ethic, nationality as well as religion. It is actually decided by where they could fulfil the greatest sense of belonging (Ross, 2012). Building a social network with other people who can share same culture is beneficial to the development of resilience (Panter-Brick and Eggeerman, 2012). This is because they can develop a cultural relationship by supporting and understanding to each other so that they can recover from the pains caused by difficulties.

For refugees, their traumatic experience is complex. Their situations are caused by different reasons, including war, political persecution, racial oppression as well as economic sanctions. The consequences of these traumatic experiences of refugees could last for a long time, including low confidence, isolation, trauma, PTSD, depression, anxiety, substance abuse and even suicidal behaviour (Kinzie, 2007). Many researches could prove that these negative effects could be relieved if they can engage in social activities with people who come from the same cultural backgrounds (Salzman and Halloran, 2004). This is because it is easier for them to talk about their traumatic experience and any mental health issues with people who have same cultural backgrounds compared with professionals. This means that people tend to believe and trust other with same belief and cultural systems without prejudice, stigma as well as discrimination. It is a fact that these listeners are actually do not have any related professional knowledges. However, confiding is actually beneficial to the recovery from traumatic experience (Salzman and Halloran, 2004).

Refugees could gradually recover from the trauma if they could improve resilience by reacquiring the sense of belonging and being understood in the new country (Alcock, 2003). In detail, cultural shock and poor living conditions are two main difficulties after they arrive in a new country. At the beginning, they may come to a new place with huge hope of a new dreamful life in future. However, language barriers and low employment could lead to cultural isolation, which means that they are difficult to find friends who come from the same cultural backgrounds. Thus, as a result of cultural isolation, they lack of strong sense of belonging extremely.

For most refugees, they want to adapt themselves to the new country in terms of culture, which is called acculturation. During this process, their original cultural identity will change with the process of absorbing new culture. As a consequence, acculturation will destroy the completeness of original cultural system, which can lead to anxiety, trauma as well as stress to some extent (Matsuoka, 1990). Refugees have a higher possibility of being influenced by acculturation because they leave their countries caused by unexpected external forces 
involuntarily (Matsuoka, 1990).

Every culture has their own cultural practice to improve their cultural identity, which could cope with trauma and improve resilience (Crane, 2010). Cultural practices could be any traditional festivals, ceremonies as well as traditional arts. Moreover, food, music as well as drawing could be viewed as the most general cultural practices which people could engage in. If they could engage more these cultural practice with people who can share same cultural origins, they can express and understand each other without discrimination and stigma, which could be regarded as a supportive social engagement. Under this kind of comfortable and trustful environment, refugees could reacquire cultural identity and emotional regulation by decreasing the shame and embarrassment. Thus, they can express their ideas of previous experience and trauma, which is a process of exposure. This process is good for the development of resilience.

\subsection{Issues Need to Be Considered}

Basically, there are four issues, which needs to be considered, including safe environment, social networks, emotional regulation and distress toleration as well as empowerment based on strengths.

Firstly, creating a comfortable environment for them and building trustful relationship with them is important. According to client-centred practice, empathy, genuineness as well as unconditional positive regard could develop a trustful relationship between social workers and clients, which could be viewed as the foundation of help clients to develop their ability of self-determination and self-management (Levant and Shlien, 1984). For example, in order to use this practice, social workers and professionals must respect clients' all previous experience for developing an equal and solid relationships between clients and social workers (Grant, 1990). By this way, refugees may gradually feel more comfortable with social workers, which is the precondition for the further interventions. In addition, it is important to give enough time for clients to respond if social workers want to use client-centred theory (Grant, 1990). In detail, this could allow clients to find their own way in their own time instead of being forced to talk by professionals.

Secondly, it is important to rebuild their social networks. Many researches have proved that developing a positive social connection could support each other, which is helpful to develop resilience (Joseph, 1999). According to social learning theory, people could learn behaviour through observation and imitation so that they could have better abilities to adapt to environment (Scherer, Adams and Wiebe, 1989). Thus, more social engagement could let them understand how to react the such environmental change effectively. During the process of communication and social activities, they could share information and make new friends. For most refugees, they always live alone without friends and family members who share similar cultural background. Thus, it is positive for them to decrease the feeling of isolation if they could try to spend more time with friends.

Thirdly, it is important to learn how to regulate personal emotions and tolerate distress for refugees. To some extent, this is also related to resilience. Resilience is used to describe a person's ability of deal with tough times and unexpected changes in life by using personal strengths or seeking help from personal social networks (Garavan and Albaugh, 2019). Basically, the first step of emotional regulation is to observe and identify the emotions. At the same time, clients could have the ability of endure the negative impacts of emotions instead of being overwhelmed by these negative emotions. Learning console themselves is important, which means that clients could calm themselves and reduce negative impacts of emotions by decreasing emotional intensity (Morawska, Dittman and Rusby, 2019). There are a lot of ways to comfort themselves, including reading, drawing, listening to music, singing songs, climbing mountains, walking as well as doing sports. By this way, clients could develop their ability of adjusting emotions instead of showing overreaction or self-harm behaviours (Galatzer-Levy, Burton and Bonanno, 2012). The following step is to accept personal emotions and feelings, which is the unreplaceable part of recovery. This means that refugees could admit the exist of these negative emotions (Gilbert, 2002). Based on this idea, they could live with these negative simultaneously. In other words, clients could have enough ability of allowing themselves to feel the exist of these emotions. This requires clients to learn the skill of distress tolerance. Similarly, this also need the acceptance and endurance of possible negative emotions. Moreover, clients should learn some useful skills to help them endure negative emotions effectively. Specifically, it is better for refugees that they could learn to accept themselves, including all previous experience, emotional uncomfortable as well as all difficulties without unreasonable judgement. One important point cannot be ignored. That is accepting these negative emotions is different with approving these exist forever (Corcoran, Farb, Anderson and Segal, 2009). The fact is that clients could learn how to tolerate these negative emotions and difficult situations. This is because distress tolerance could help refugees to develop their resilience (Correia, Neves and Verissimo, 2004).

Fourthly, improving refugees' resilience based on strength theory and empowerment theory simultaneously. 
Strength theory focuses on clients' capacities and skills internally and externally at the same time (Rashid, 2015). Clients are no longer viewed as a person who needs help. However, they could be treated as an expert in some specific areas so that social workers and clients could make a joint effort to improve clients' confidence, self-determination as well as responsibilities collaboratively (Rashid, 2015). This is because clients could unleash their potential abilities. Similarly, empowerment theory pays more attention to help clients learn how to control the resources around them effectively by improving individual's skills based on personal strengths (Gibson, 1993). It is same with strength theory that social workers are facilitators instead of leaders. In most conditions, social workers and professionals could use these two theories at the same time. This is because they all focus on the positive aspects of clients instead of negative things and difficulties faced by clients (Guo and Tsui, 2010). Thus, they all advocate that clients should ignore negative aspects and build ability of solving problems (Guo and Tsui, 2010). By this way, refugees could decrease the possibility of recalling the miserable experience, which could decrease the impacts of negative emotions and mental health problems (Førde, 2007).

In general, social workers should take at least four elements into consideration. These four issues are creating a safe environment for trust, rebuilding individual's social networks, improving their ability of tolerate negative emotions and controlling emotions as well as using strength-based theory and empowerment theory.

\subsection{Intervention Approaches}

Basically, there are three basic and popular intervention approaches which are always used in coping with trauma in refugee population, including cognitive-behaviour therapy, psychoeducation and parenting intervention as well as art therapy.

Firstly, cognitive-behaviour therapy (CBT) is a useful trauma-informed treatment for refugee children who is experiencing trauma. CBT advocates that what we behave is decided by how we see and interpret the world (Otto and Hinton, 2006). Thus, based on this thought, social workers believe that reasonable behaviours can be rebuilt as long as thoughts could be changed by teaching because behaviours are decided by thoughts (Otto and Hinton, 2006). In order to adjust CBT to children's situation, school-based CBT interventions are developed. This is designed to deal with children who have mental health issues, such as depression, anxiety, trauma as well as PTSD (Bernstein, Bernat, Vioctor and Layne, 2008). Thus, this could be useful for refugees because it could focus on trauma and cultural shock at the same time (Bernstein, Bernat, Vioctor and Layne, 2008). In general, this intervention needs about ten sessions. At the first stage, refugee children need to finish a self-report questionnaire to figure out all signs and symptoms of PTSD and trauma. At the following steps, social workers and related professionals could provide treatments within school settings. Schools are a relatively stable and safe environment for refugee children. Thus, they could receive treatments more effectively while it is easy for social workers to have observations and assessment in terms of their behaviours and thoughts.

Secondly, for refugee mothers, psychoeducation and parenting intervention should be provided. This approach is aimed of maintain the mental health of mothers and their refugee children at the same time (Schottelkorb, Doumas and Garcia, 2012). Basically, mothers and children are taught to be given an educational schedule lasting about five months. During this period, basic medical care, therapeutic discussions as well as psychoeducation are processed in scheduled group meetings with necessary ongoing evaluations. More importantly, useful guidance about parent-child interactions and communication is provided, which focuses on decreasing the negative impacts between each other. This is because negative impacts will increase manyfold if children and mothers could not maintain a stable emotion when they are living together. Based on this explanation, child parent relationship therapy (CPRT) could be used for them. CPRT is a treatment based on play, which could improve their emotional and attachment ties between each other (Carnes-Holt and Bratton, 2014). In such supportive group environment, children could learn positively in terms of behaviours and emotions from their parents while parents could learn how to meet children's needs of love, safety as well as acceptance (Kidron and Landreth, 2010). Thus, children could improve their well-being by decreasing the negative impacts of behavioural, emotional, social and attachment disorders. CPRT could be supported by attachment theory. Attachment theory emphasizes on the importance of attachment bond between child and caregivers. The main argument of this theory is that child will seek security and attachment from caregivers in nature. If their attachment is positive, child could develop their ability of solving problems and confidence by observing and interacting with environment (Kennedy and Kennedy, 2004). Thus, based on CPRT and attachment theory, refugee children and parents should deserve a comfortable and safe environment in order to rebuild a solid attachment relationship. By this way, parents and children could improve their skills of coping with negative emotions simultaneously.

Thirdly, art therapy could be used when they are reluctant to expression. In detail, for some people with trauma, 
they can show highly resistant and embarrassment so that they always can not use language and facial expressions to show their feelings. Therefore, art should be viewed as a useful way to show personal feelings (Linesch, 2013). For example, some refugees think that talking about traumatic experience will make them more painful so that they do not want to talk their previous experience with social workers and other professionals. Compared with talking, art is a relatively less threatening way to express ideas for them. In addition, refugees could fulfil the need of counterbalance to their losses by getting the sense of control and feeling of structure (Kalmanowitz, 2016). However, it is necessary to consult with refugees before using art therapy because art therapy may deteriorate clients' situation (Talwar, 2007). In detail, during the process of creating artworks, clients will recall their previous experience because they know that this is a kind of intervention, which could influence the positive effects of art therapy.

In conclusion, CBT, psychoeducation and parenting intervention as well as art therapy are always found in dealing with trauma among refugees.

\subsection{Reflection}

After writing this essay, some new knowledge and practice advice are acquired, which is useful for social work students.

First of all, building a comfortable and trustful relationship with clients in terms of cultural safety and interventional environment. This is the precondition for all further actions, which can improve the efficiency of all intervention approaches.

Secondly, it is necessary to understand the relationship between brain and experience. Actually, brain development could be influenced by experience (Carrion and Wong, 2012). Thus, as a professional social worker, it is reasonable that clients should be told the information about trauma, its impacts and how personal experience will influence the trauma developed in brain. This kind of education is one important step in therapy because clients have the right to understand themselves.

Thirdly, all interventions should be changed according to different situations of clients. Social workers can not wish that clients could adapt themselves to different intervention approaches. In contrast, clients will show different responses in terms of acceptance, resilience as well as recovery (Tickle-Degnen, 1998). Therefore, social workers should have the ability change these intervention approaches more suitable for different clients in order to get more benefits.

Fourthly, it is necessary to cope with various client responses, such as reluctant to engagement, embarrassment as well as shame. Social workers should understand different clients could show different levels of self-regulation and self-integrity. Thus, social workers should change intervention plan slightly according to client responses. For example, when one client shows serious shame or embarrassment, these feelings refer to her selves or he selves instead of any external influences. Thus, social workers may should add the recovery of the sense of a damaged to the main intervention plan.

Fifthly, rebuilding the personal social networks should be emphasized on the intervention plan. This is because social networks could provide positive supports for the recovery from trauma.

Sixthly, it is a fact that different clients will show different levels of recovery even though same interventions are provided. This is because different clients will show different abilities of engagement, acceptance as well as recovery capability (Chao, Steffen and Heiby, 2012).

Seventhly, it is necessary to empower clients with the strength-based theory. This means that clients could focus on what they are good at instead of negative emotions, which could improve their ability of self-recovery by using the resources around them.

Eighthly, improving the ability of controlling emotions and self-regulation should be taught as the foundation. This is because clients could improve their safety if they can manage their negative emotions (Croskerry, Abbass and $\mathrm{Wu}, 2010)$.

Ninthly, regularly and continually supervision is necessary for interventions. This is because social workers should control the process and different sessions of the whole intervention plan. In different stages, assessment and supervision should be processed in order to clarify the outcomes and change intervention plan slightly.

\section{Conclusion}

This essay explains why examining refugees' experience is important in terms of their contributions and negative impacts brought in a new country and their mental health problems caused by traumatic experience. Then, low cultural identity is one factor contributing to the trauma of refugees. In addition, some issues should be considered when making interventions, including building a trustful and comfortable environment, establishing supportive social networks, combining strength theory and empowerment theory for intervention plans as well as improving their ability of toleration and emotional regulation. Thus, CBT, psychoeducation and parenting 
intervention as well as art therapy could be considered to apply for refugees' trauma. Lastly, some reflections are concluded from writing this essay.

\section{References}

Alcock, M. (2003). Refugee trauma - The assault on meaning. Psychodynamic Practice, 9(3), 291-306.

Bernstein, G. A., Bernat, D. H., Victor, A. M., \& Layne, A. E. (2008). School-based interventions for anxious children: 3-, 6-, and 12-month follow-ups. Journal of the American Academy of Child \& Adolescent Psychiatry, 47(9), 1039-1047.

Carnes-Holt, K., \& Bratton, S. C. (2014). The efficacy of child parent relationship therapy for adopted children with attachment disruptions. Journal of Counseling \& Development, 92(3), 328-337.

Carrion, V. G., \& Wong, S. S. (2012). Can traumatic stress alter the brain? Understanding the implications of early trauma on brain development and learning. Journal of Adolescent Health, 51(2), S23-S28.

Chao, P. J., Steffen, J. J., \& Heiby, E. M. (2012). The effects of working alliance and client-clinician ethnic match on recovery status. Community Mental Health Journal, 48(1), 91-97.

Coats, D. R. (2017). Worldwide Threat Assessment.

Corcoran, K. M., Farb, N. A., Anderson, A., \& Segal, Z. (2009). Mindfulness and emotion regulation. Emotion Regulation and Psychopathology, 339-355.

Correa-Velez, I., Gifford, S. M., \& Barnett, A. G. (2010). Longing to belong: Social inclusion and wellbeing among youth with refugee backgrounds in the first three years in Melbourne, Australia. Social Science \& Medicine, 71(8), 1399-1408.

Correia, M., Neves, N. F., \& Verissimo, P. (2004, October). How to tolerate half less one Byzantine nodes in practical distributed systems. In Proceedings of the 23rd IEEE International Symposium on Reliable Distributed Systems (pp. 174-183). IEEE.

Crane, T. A. (2010). Of models and meanings: cultural resilience in social-ecological systems. Ecology and Society, 15(4), 19-19.

Croskerry, P., Abbass, A., \& Wu, A. W. (2010). Emotional influences in patient safety. Journal of Patient Safety, 6(4), 199-205.

Dalton-Greyling, T., \& Campus, A. P. (2008, October). Urban refugees: Definitions, legal positions and wellbeing in South Africa. In Unpublished paper presented at the Annual Forum of the Trade \& Industrial Policy Strategy (pp. 27-31). Cape Town, South Africa.

De Vroome, T., \& Van Tubergen, F. (2010). The employment experience of refugees in the Netherlands. International Migration Review, 44(2), 376-403.

Dembo, R., La Voie, L., Schmeidler, J., \& Washburn, M. (1987). The nature and correlates of psychological/emotional functioning among a sample of detained youths. Criminal Justice and Behavior, 14(3), 311-334.

Dudley, M. (2003). Contradictory Australian national policies on self-harm and suicide: The case of asylum seekers in mandatory detention. Australasian Psychiatry, 11(sup1), S102-S108.

Ferrada-Noli, M., \& Sundbom, E. (1996). Cultural bias in suicidal behaviour among refugees with post-traumatic stress disorder. Nordic Journal of Psychiatry, 50(3), 185-191.

Førde, S. (2007). Refugee children and resilience: empowerment, participation and subjective wellbeing. Master's thesis.

Galatzer-Levy, I. R., Burton, C. L., \& Bonanno, G. A. (2012). Coping flexibility, potentially traumatic life events, and resilience: A prospective study of college student adjustment. Journal of Social and Clinical Psychology, 31(6), 542-567.

Garavan, H., \& Albaugh, M. (2019). Connecting With Resilience. Biological Psychiatry, 85(8), 621-622.

Gibson, C. M. (1993). Empowerment theory and practice with adolescents of color in the child welfare system. Families in Society, 74(7), 387-396.

Gilbert, M. (2002). Collective guilt and collective guilt feelings. The Journal of Ethics, 6(2), 115-143.

Grant, B. (1990). Principled and instrumental non-directiveness in person-centered and client-centered therapy. Person-Centered Review, 5(1), 77-88.

Guo, W. H., \& Tsui, M. S. (2010). From resilience to resistance: A reconstruction of the strengths perspective in social work practice. International Social Work, 53(2), 233-245.

Joseph, S. (1999). Social support and mental health following trauma. 
Kalmanowitz, D. (2016). Inhabited studio: Art therapy and mindfulness, resilience, adversity and refugees. International Journal of Art Therapy, 21(2), 75-84.

Karlsen, E. (2016). Refugee resettlement to Australia: what are the facts?.

Kennedy, J. H., \& Kennedy, C. E. (2004). Attachment theory: Implications for school psychology. Psychology in the Schools, 41(2), 247-259.

Kidron, M., \& Landreth, G. (2010). Intensive child parent relationship therapy with Israeli parents in Israel. International Journal of Play Therapy, 19(2), 64.

Kinzie, J. D. (2007). PTSD Among Traumatized Refugees.

Kok, W. (1989). Self-settled refugees and the socio-economic impact of their presence on Kassala, Eastern Sudan. Journal of Refugee Studies, 2(4), 419-440.

Levant, R. F., \& Shlien, J. M. (1984). Client-centered therapy and the person-centered approach: New directions in theory, research, and practice. Praeger Publishers/Greenwood Publishing Group.

Linesch, D. G. (2013). Art therapy with families in crisis: Overcoming resistance through nonverbal expression. Routledge.

Loomes, S., \& McCarthy, G. (2011). The aging workforce: how can Australian universities address future workforce challenges?

Matsuoka, J. K. (1990). Differential acculturation among Vietnamese refugees. Social Work, 35(4), 341-345.

Momartin, S., Silove, D., Manicavasagar, V., \& Steel, Z. (2004). Comorbidity of PTSD and depression: associations with trauma exposure, symptom severity and functional impairment in Bosnian refugees resettled in Australia. Journal of Affective Disorders, 80(2-3), 231-238.

Morawska, A., Dittman, C. K., \& Rusby, J. C. (2019). Promoting Self-Regulation in Young Children: The Role of Parenting Interventions. Clinical Child and Family Psychology Review, 22(1), 43-51.

Otto, M. W., \& Hinton, D. E. (2006). Modifying exposure-based CBT for Cambodian refugees with posttraumatic stress disorder. Cognitive and Behavioral Practice, 13(4), 261-270.

Panter-Brick, C., \& Eggerman, M. (2012). Understanding culture, resilience, and mental health: The production of hope. In The social ecology of resilience (pp. 369-386). Springer, New York, NY.

Phillips, J., \& Spinks, H. (2013). Boat arrivals in Australia since 1976. Parliament of Australia, Department of Parliamentary Services, Parliamentary Library.

Rashid, T. (2015). Positive psychotherapy: A strength-based approach. The Journal of Positive Psychology, 10(1), 25-40.

Ross, M. H. (Ed.). (2012). Culture and belonging in divided societies: Contestation and symbolic landscapes. University of Pennsylvania Press.

Salzman, M. B., \& Halloran, M. J. (2004). Cultural trauma and recovery: Cultural meaning, self-esteem, and the reconstruction of the cultural anxiety buffer. Handbook of Experimental Existential Psychology, 231-246.

Scherer, R. F., Adams, J. S., \& Wiebe, F. A. (1989). Developing entrepreneurial behaviours: A social learning theory perspective. Journal of Organizational Change Management, 2(3), 16-27.

Schottelkorb, A. A., Doumas, D. M., \& Garcia, R. (2012). Treatment for childhood refugee trauma: A randomized, controlled trial. International Journal of Play Therapy, 21(2), 57.

Smith, L. (1998). Behavioral and emotional characteristics of children in detention. Journal of Correctional Education, 63-66.

Talwar, S. (2007). Accessing traumatic memory through art making: An art therapy trauma protocol (ATTP). The Arts in Psychotherapy, 34(1), 22-35.

Tickle-Degnen, L. (1998). Using research evidence in planning treatment for the individual client. Canadian Journal of Occupational Therapy, 65(3), 152-159.

\section{Copyrights}

Copyright for this article is retained by the author(s), with first publication rights granted to the journal.

This is an open-access article distributed under the terms and conditions of the Creative Commons Attribution license (http://creativecommons.org/licenses/by/4.0/). 